\title{
AVALIAÇÃO DA ASSISTENCIA PRESTADA EM UM CENTRO ASSISTENCIAL DE ENFERMAGEM *
}

\author{
Angela Maria Geraldo Pierin** \\ Cilene Aparecida Costardi Ide** \\ Miako Kimura** \\ Vera Lúcia Conceição de Gouveia Santos ***
}

PIERIN, A.M.G.; IDE, C.A.C., KIMURA, M.; SANTOS, V.L.C. de G. Avaliação da assistência prestada em um Centro Centro Assistencial de Enfermagem. Rev. Esc. Enf. USP, São Paulo, 20(1):71-82, 1986.

As autoras apresentam os resultados da avaliação da assistência de enfermagem prestada por um Centro organizado pelas docentes de Enfermagem MédicoCirürgica da Escola de Enfermagem da USP, que representa uma atividade de extensão de serviços à comunidade, corsstituida pelo servidores da Escola. Essa avaliação foi feita pelas docentes envolvidas no projeto e pela clientela atendida. Ambas relataram satisfação quanto à assistência recebida e prestada e apresentaram sugestões para aprimoramento do mesmo.

\section{INTRODUÇÃO}

Em 1983 foi criado, na Escola de Enfermagem da USP (EEUSP), o Centro Assistencial de Enfermagem (CAE), vinculado à Disciplina de Enfermagem Médico-Cirúrgica (EMC). Este um centro de extensão de serviços à comunidade, desenvolvido pelas docentes da referida disciplina, teve como principal objetivo prestar assistência de enfermagem à população da EE com base nos problemas identificados, valendo-se também dos recursos de saúde existentes na comunidade.

Decorridos nove meses após a instalação, foi preocupação das docentes, avaliar 0 atendimento prestado, com a participação dinâmica da clientela e das próprias docentes. Considerou-se relevante, ainda, a quantificação das atividades desenvolvidas neste periodo, o que possibilitaria mensurar o desempenho do grupo em face dos objetivos propostos.

Esta avaliação deveria retratar, principalmente, a percepção da clientela acerca do atendimento recebido, bem como o conhecimento de suas expectativas e reações, a serem utilizados, posteriormente, como subsidios para o aprimoramento da assistência prestada.

* Trabalho apresentado no XXXVI Congresso Brasileiro de Enfermagem - Belo Horizonte, 1984.

** Enfermeira. Mestre em enfermagem. Professor Assistente do Departamento de Enfermagem Médico-Cirárgica da Escola de Enfermagem da USP - disciplina Infermagem Médico-Cirúreica.

*** Enfermeira. Auxillar de Ensino do Departamento de Enfermagem Médico-Cirúrgica da Escola de Enfermagem da USP - disciplina Enfermarem MGdioo-Cirtirxica. 
Além disso, é meta do grupo desenvolver na população assistida o compromisso com a própria saúde, encorajando a sua participação a partir de sugestões e propostas para a adequação do trabalho desenvolvido no CAE.

Em vista do exposto, julgou-se importante a realização deste trabalho, que tem como objetivos: no CAE.

- quantificar o atendimento desenvolvido pelas docentes de EMC

- avaliar o atendimento prestado no CAE, a partir da opinião da clientela e das docentes de EMC.

\section{METODOLOGLA}

O presente estudo foi realizado no CAE da EEUSP.

\section{População}

A população foi constituída por 37 funcionários da EEUSP assistidos no CAE, que satisfizeram aos critérios de inclusão, e pelas 12 docentes de EMC que prestaram atendimento neste serviço.

Foram fixados os seguintes critérios para inclusão na população: de 1984,

- clientes atendidos no CAE no período de junho de 1983 a março

- clientes que responderam ao formulário de avaliação e

- docentes que participaram da assistência prestada no CAE.

\section{Coleta de dados}

A coleta de dados foi realizada no mês de março de 1984, com base em instrumento especifico (ANEXO I).

\section{Instrumento .}

Para a quantificação do atendimento prestado, procedeu-se à somatória das diferentes atividades exercidas, mediante utilização de formulário específico (ANEXO II).

O instrumento utilizado para a avaliação foi constituído por perguntas fechadas e abertas que possibilitaram ao cliente e à docente localizarem a sua opinião sobre o atendimento.

2. Operacionalização da coleta de dados

A quantificação do atendimento, assim como a distribuição do formulário de avaliação, foram realizados pelas docentes que participaram do atendimento. Estabeleceu-se o prazo de uma semana para a devolução do impresso de avaliação.

\section{Tratamento estatístico}

Os resultados obtidos serão analisados em freqüências absolutas e relativas. 


\section{RESULTADOS}

Os resultados obtidos serão apresentados e analisados por meio de avaliação quantitativa e qualitativa.

1. Avaliação quantitativa das atividades desenvolvidas no CAE.

Os resultados expressos a seguir caracterizam a assistência prestada pelas docentes da disciplina de EMC no CAE, incluindo encaminhamentos e atendimentos eventuais.

É necessário esclarecer que, para a efetivação dessas atividades, utilizaram-se recursos dentro e fora da EEUSP. Em âmbito interno contou-se com a colaboração das docentes da disciplina de Nutrição Aplicada à Enfermagem, que atendem no Centro de Assistência Nutricional, e a participação de docentes das seguintes disciplinas: Enfermagem em Centro Cirúrgico, Enfermagem em Doenças Transmissíveis, Enfermagem Ginecológica e Obstétrica, Enfermagem Pediátrica, Enfermagem Preventiva e Comunitária e Enfermagem Psiquiátrica. Estas docentes colaboraram prestando assistência aos clientes encaminhados pelo CAE, dentro de sua competência.

Estabeleceram-se também convênios com recursos da comunidade, expressos a seguir:

- Centro de Imunizações do Hospital das Clínicas da Faculdade de Medicina da USP (HC FMUSP), para a inclusão do adulto num esquema de vacinação adequado;

- Centro de Saúde Geraldo Paula Souza, da Faculdade de Saúde Pública da USP, para o atendimento de diversos problemas de saúde, inclusive oftalmológicos;

- Instituto Adolfo Lutz, para o controle periódico de exames protoparasitológicos dos funcionários que manipulam alimentos e aqueles com sintomatologia específica;

- Fundação Centro de Pesquisa em Oncologia de São Paulo, para a realização do controle ginecológico da população feminina;

- Hospital do Servidor Público Estadual, para atendimento dos funcionários com direito a tratamento de saúde nesta instituição;

- Ambulatório de Dermatologia, Pronto Socorro e Liga de Diagnóstico e Tratamento da Hipertensão Arterial, todos do HC FMUSP, para os tratamentos de problemas de sua competência.

O processo de atendimento, propriamente dito, abrangeu: consultas de enfermagem (primeira e subseqüentes) e atendimentos eventuais a problemas específicos de saúde, que necssitam de implementação de cuidados de enfermagem e sem agendamento prévio. Como emergências foram consideradas as situações críticas que exigiam pronto atendimento. 
TABELLA 1

MOVIMENTO DA CLIENTELA ATENDIDA NO CAE NO PERIODO DE JUNHO DE 1983 A MARÇO DE 1984, EEUSP.

\begin{tabular}{|c|c|c|c|}
\hline Atendimentos & $\mathbf{n}^{\bullet}$ & $\%$ & \\
\hline Atendimentos eventuais e emergenciais & 96 & 47,3 & \multirow{3}{*}{52,7} \\
\hline 1.4 consulta & 64 & $31,5]$ & \\
\hline Retorno & 43 & 21,2 & \\
\hline Total & 203 & 100,0 & \\
\hline
\end{tabular}

Verifica-se pelos dados da Tabela 1 que, além das atividades progra madas que corresponderam a 52,7\% do total, foi significativo também o número de atendimentos eventuais $(47,3 \%)$. Estes fatos evidenciam a necessidade de uma programação contínua de enfermagem para a cobertura das necessidades de saúde da população.

Vale acrescentar que os 64 funcionários da instituição que passaram por $1^{\mathrm{a}}$ consulta equivalem a $73,6 \%$ do contingente total.

TABELA 2

ATENDIMENTOS REALIZADOS NO CAE NO PERIODO DE JUNHO DE 1983 A MARÇO DE 1984, EEUSP.

\begin{tabular}{lcc}
\multicolumn{1}{c}{ Atendimentos } & $\mathrm{n}^{\natural}$ & $\%$ \\
\hline Controle dos sinais vitais & 30 & 31,3 \\
Emergências & 22 & 22,9 \\
Visitas domiciliárias & 15 & 15,6 \\
Fornecimento de medicação & 10 & 10,4 \\
Orientações específicas & 9 & 9,4 \\
Aplicação de medicação parenteral & 6 & 6,3 \\
Curativos & 3 & 3,1 \\
Imobilização & 1 & 1,0 \\
\hline \multicolumn{1}{c}{ Total } & 96 & 100,0 \\
\hline
\end{tabular}

Os resultados apresentados na Tabela 2 demonstram que $31,3 \%$ do atendimento foram de controle de sinais vitais, o que caracteriza agravos à saúde da população assistida.

Outro dado relevante refere-se ao elevado número de emergências atendidas $(22,9 \%)$, o que evidencia os riscos inerentes ao trabalho a que essa população está exposta. 
TABELLA 3

ENCAMINHAMENTOS FEITOS PELO CAE, NO PERIODO DE JUNHO DE 1983 A MARÇO DE 1984, EEUSP.

\begin{tabular}{lrr}
\hline Encaminhamentos & $\mathrm{n}^{\circ}$ & $\%$ \\
\hline Para a EE & 15 & 7,6 \\
Para fora da EE & 183 & 92,4 \\
\hline Total & 198 & 100,0 \\
\hline
\end{tabular}

Os dados da Tabela 3 evidenciam uma realidade profissional: a inter dependência da equipe de saúde, uma vez que, dos problemas de enfermagem identificados, a maioria necessita de encaminhamento a locais outros que a EE para sua solução.

TABELA 4

ENCAMINHAMENTOS DOS CLIENTES DO CAE AOS RECURSOS DA COMUNIDADE, NO PERIODO DE JUNHO DE 1983 A MARÇO DE 1984, EEUSP.

\begin{tabular}{|c|c|c|}
\hline Recursos da Comunidado & $\mathbf{n}^{\circ}$ & $\%$ \\
\hline Centro de Imunizações de adultos do Hospital das Clínicas & 60 & 32,8 \\
\hline Centro de Saúde Geraldo Paula Souza & 36 & 19,7 \\
\hline Instituto Adolfo Lutz & 35 & 19,1 \\
\hline Fundação Centro de Pesquisa em Oncologia & 23 & 12,6 \\
\hline Ambulatório de Dermatologia do Hospital das Clínicas & 9 & 4,9 \\
\hline Pronto Socorro do Hospital das Clinicas & 9 & 4,9 \\
\hline Hospital do Servidor Público Estadual & 8 & 4,4 \\
\hline Liga de Hipertensão do Hospital das Clínicas & 3 & 1,6 \\
\hline Total & 183 & 100,0 \\
\hline
\end{tabular}

Os encaminhamentos para o Centro de Imunizações de adultos do Instituto Adolfo Lutz e para a Fundação Centro de Pesquisa em Oncologia fazem parte de uma programação específica do CAE: a inclusão do adulto no esquema de vacinação adequada, o controle protoparasitológico periódico de um grupo de funcionários e a prevenção de câncer ginecológico do contingente feminino, respectivamente. 
Pelos dados da Tabela 5, observa-se que, além da utilização dos recursos acima referidos, foi marcante o número de encaminhamentos ao Centro de Saúde $(19,7 \%)$, para a solução de problemas físicos identificados pelos docentes e que necessitaram de atendimento médico.

3.2. Avaliação qualitativa da assistência prestada.

Os resultados expostos a seguir referem-se à avaliação dos funcionários assistidos e das docentes de Enfermagem Médico-Cirúrgica que participaram do atendimento. Expressam, também, sugestões e expectativas sobre este serviço.

TABELA 5

JUSTIFICATTVAS DOS CLIENTES SOBRE SATISFAÇO QUANTO AO ATENDIMEINTO RECEBIDO, EEUSP, 1984.

\begin{tabular}{lrrr}
\hline \multicolumn{1}{c}{ Justificativas } & $\mathbf{n}^{\bullet}$ & $\%$ \\
\hline Receberam bom atendimento & 10 & 37,0 \\
Atendimento ajudou a identificação de problemas de saúde & 9 & 33,3 \\
Facilitou ao acesso a outros recursos de saúde & 8 & 29,7 \\
\hline & Total & 27 & 100,0 \\
\hline
\end{tabular}

Dos funcionários assistidos no CAE no periodo de junho de 1983 a março de 1984 e que responderam ao formulário de avaliação, a maioria $(97,3 \%)$ referiu satisfação quanto ao atendimento recebido e apenas 1 funcionário $(2,7 \%)$ manifestou insatisfação, não esclarecendo a causa. Dos satisfeitos, 27 justificaram os seus motivos, os quais se encontram expressos na tabela acima.

TABEHA 6

ASPECTOS POSTIVOS DO ATENDIMENTO, SEGUNDO OS CLIENTES, EHEUSP, 1984.

\begin{tabular}{lrrr}
\hline \multicolumn{1}{c}{ Aspectos Positivos } & n & \% \\
\hline Recebeu atenção durante o atendimento & 14 & 82,4 \\
Atendimento não sujeito a espera & 2 & 11,8 \\
Recebeu orientação pertinente & 1 & 5,8 \\
\hline Total & 17 & 100,0 \\
\hline
\end{tabular}

Não houve expressão de desagrado por parte da clientela atendida e sim referência a aspectos positivos sobre a assistência recebida, principalmente pelo contacto humano e atendimento individualizado, conforme os resultados da Tabela 7. 

SUGESToES PROPOSTAS PELOS CLIENTES PARA A MELHORIA
DO ATENDIMENTO, EEUSP, 1984.

\begin{tabular}{lccc}
\hline \multicolumn{1}{c}{ Sugestões } & $\mathbf{n}^{9}$ & $\%$ \\
\hline Encaminhamento para tratamento odontológico & 16 & 66,6 \\
Doação dos medicamentos prescritos pelos médicos & 5 & 20,8 \\
Outros : & 3 & 12,6 \\
\hline Total & 24 & 100,0 \\
\hline
\end{tabular}

Outros: aula de ginástica, atendimento a familiares e outros convênios com o Hospital das Clínicas.

As sugestões expressas na Tabela 8 caracterizam deficiência dos recursos da comunidade para o atendimento de problemas de saúde prioritários da clientela: tratamento odontológico e aquisição de medicamentos, os quais não são incluídos no atendimento recebido no CAE.

TABELA 8

JUSTIFICATIVAS DAS DOENTES SOBRE SATISFAÇAO QUANTO AO ATENDIMENTO PRESTADO, EEUSP, 1984.

\begin{tabular}{lccc}
\hline & Justificativas & $n^{\circ}$ & $\%$ \\
\hline $\begin{array}{l}\text { Possibilita desenvolvimento de assistência de enfermagem } \\
\text { autônoma, sistematizada e abrangente }\end{array}$ & 12 & 48,0 \\
Possibilita contacto interpessoal com os clientes e os motiva & 10 & 40,0 \\
Outros & Total & 3 & 12,0 \\
\hline
\end{tabular}

Foi unânime a referência de satisfação por parte das docentes quanto ao trabalho desempenhado no CAE, conforme os dados da tabela 9, principalmente por assistir à população da EE, possibilitando atendimento individualizado que abranja aspectos de prevenção das doenças e promoção e recuperação da saúde.

Foi satisfatória, também, a realização de um trabalho em equipe multiprofissional a partir dos recursos contactados dentro e fora da instituição. 
TABELA 9

SUGESTÕES PROPOSTAS PELAS DOCENTES PARA MELHORIA DO ATENDIMENTO.

\begin{tabular}{|c|c|c|}
\hline Sugestões & ne & $\%$ \\
\hline Ampliar o número de convênios internos e externos & 16 & $\mathbf{4 4 , 5}$ \\
\hline Revisar aspectas organizacionais & 14 & 38,9 \\
\hline Efetuar programa educativo & 4 & 11,1 \\
\hline Outros : & 2 & 5,5 \\
\hline Total & 36 & 100,0 \\
\hline
\end{tabular}

Outros: atendimento a familiares de funcionários e adequação do horário da consulta ao do cliente.

As sugestões das docentes, expressas na Tabela 9, evidenciam a neces sidade de aprimoramento do CAE no que se refere à estrutura organizacional, incluindo revisão dos impressos e controle de retornos, do material, equipamento e dos serviços externos, além da ampliação dos convênios, principalmente o odontológico.

As docentes opinaram, ainda, sobre a importância da elaboração de um programa de atividades discentes, e de cursos de orientação à saúde para funcionários, que atenderiam a um dos objetivos iniciais do CAE, em relação ao ensino.

\section{DISCUSSÃO}

A implantação do Centro Assistencial de Enfermagem, como uma extensão de serviços à comunidade, representou um desafio para as docentes da disciplina de enfermagem Médico-Cirúrgica. O compromisso assumido por todās, representou uma ampliação na assistência preconizada pelo grupo, na medida em que desenvolveu uma assistência de Enfermagem abrangendo aspectos de prevenção da doença e promoção e recuperação da Saúde. Significou também a viabilidade de um trabalho conjunto entre as diversas disciplinas da Escola de Enfermagem, além de um trabalho multiprofissional, com a utilização de diferentes recursos da Comunidade. Outro aspecto relevante desse serviço foi a proposta de atuação junto a uma população até então habituada apenas a procurar atendimento à saúde esporádico e essencialmente curativo. Finalmente, a implantação do CAE constituiu uma tentativa de desenvolver o exercicio autônomo da profissão.

Uma vez o CAE organizado e implantado, decorridos 9 meses de atendimento, sentiu-se a necessidade de avaliação que caracterizasse a assistência prestada, a partir do grupo que desenvolveu essa atividade, como também dos clientes que a receberam. 
Os resultados obtidos nesse processo avaliativo demonstram que o serviço desenvolvido no CAE abrangeu $73,6 \%$ do contingente de funcionários da instituição (tabela 1). Além da assistência programada foi significativo o número dos atendimentos eventuais e de emergências $(47,3 \%)$. Este fato justifica não somente a necessidade de atividade sistematizada e contínua, como também a relevância de programas de orientação à saúde, inclusive de prevenção de acidentes.

Dentre os atendimentos eventuais caracterizados na tabela 2 , houve predomínio do controle de sinais vitais, confirmando o comprometimento físico desses funcionários, fator que provavelmente interfere no trabalho por eles desempenhado.

A assistência prestada no CAE (tabela 3), retrata a interdependência multiprofissional uma vez que, $92,4 \%$ dos encaminhamentos foram para serviços fora da EE. Dentre estes, foi significativo o número de encaminhamentos ao Centro de Saúde $(19,7 \%)$, por queixas físicas ou sintomatológicas identificadas, caracterizando uma população doente. Dos demais encaminhamentos os mais freqüentes foram para o Centro de Imunização (32,8\%), Instituto Adolfo Lutz (19,1\%) e Fundação Centro de Pesquisa em Oncologia $(12,6 \%)$, que fazem parte de uma programação específica e profilática (tabela 4). Os dados expressos nas tabelas 3 e 4, demonstram que para assistir à saúde é indispensável um trabalho multiprofissional em equipe, uma vez que, a partir dos problemas identificados pelas enfermeiras do $\mathrm{CAE}$, foi significativo o número de encaminhamentos a outros serviços, para sua solução.

No que se refere à avaliação qualitativa da assistência prestada, verifica-se que $97,3 \%$ dos funcionários expressaram satisfação, principalmente por terem recebido bom atendimento, por este ter ajudado a identificar problemas de saúde e por ter facilitado o acesso a outros recursos da Comunidade (tabela 5).

A clientela assistida ressaltou, como aspectos positivos do atendimento, a atenção recebida, não sujeita à espera. Isto retrata a satisfação que o atendimento individualizado e humanizado propicia ao assistido (tabela 6).

As sugestões propostas pelos funcionários reforçam a necessidade da ampliação dos convênios, abrangendo principalmente o atendimento odontológico e a doação de medicamentos. O fornecimento de remédios não está incluído nas atividades do CAE, porém, a partir dessa referência, sentiu-se a necessidade de se tentar solucionar tal problema, fornecendo-os quando possivel, mediante a apresentação da receita médica.

As docentes da disciplina de EMC que atuaram no CAE, demonstraram, unanimamente, satisfação quanto ao atendimento prestado. Justificaram este sentimento pela possibilidade de desenvolverem assistência de enfermagem sistematizada, abrangente, autônoma e dirigida para o auto-cuidado, além de ter essa atividade possibilitado relacionamento interpessoal (tabela 8). Quanto ao atendimento odontológico, estabeleceu-se contato com entidades visando este tipo de serviço. 
As sugestōes propostas pelas docentes incluem a ampliação de recursos que solucionem os problemas de saúde prioritários da clientela, além da revisão de aspectos organizacionais para o aprimoramento do serviço. As docentes manifestaram, também, a premência de se desenvolverem programas educativos que abranjam atividades discentes, além de cursos de orientação à saúde para a população, os quais efetivaram um dos objetivos iniciais do CAE, relacionado ao ensino (tabela 9).

\section{CONCLUSOEES}

Os resultados da avaliação quantitativa da assistência prestada no CAE, no período de junho de 1983 a março de 1984, conduziram às seguintes conclusões:

- foram realizados 203 atendimentos, dos quais eventuais e de emergência $(47,3 \%)$, primeira consulta $(31,5 \%)$ e retornos $(21,2 \%)$;

- dentre os 198 encaminhamentos realizados, 92,4\% foram para serviços fora da EE.

No que se refere à avaliação qualitativa verificou-se que:

- a maioria da clientela $(97,3 \%)$ referiu satisfação quanto ao atendimento recebido;

- os aspectos positivos referidos pelos funcionários valorizaram o contato humano e o atendimento individualizado recebido;

- as sugestões propostas pelos clientes abrangeram o encaminhamento a um serviço odontológico $(66,6 \%)$ e a doação de medicamentos prescritos (pelo médico) $(20,8 \%)$;

- a totalidade dos docentes referiu satisfação quanto ao trabalho desenvolvido no CAE;

- os principais aspectos positivos ressaltados pelos professores que atuam no CAE dizem respeito à possibilidade de desenvolverem e efetivarem assistência de enfermagem sistematizada, abrangente, autônoma e dirigida para o auto-cuidado;

- as sugestoes propostas pelos docentes são relativas a ampliação de convênios com outras instituições de saúde, além da revisão de aspectos organizacionais intrínsecos ao CAE.

Os resultados acima descritos ressaltam a importância do atendimento desenvolvidos bem como a validade do uso de um processo avaliativo que forneça subsídios para o aperfeiçoamento da assistência de enfermagem prestada.

PIERIN, A.M.G.; IDE, C.A.C.; KIMURA, M.; SANTOS, V.L.C. de G. Evaluation of the assistance given by a Nursing Assistance Center. Rev. Esc. Enf. USP, São Paulo, 20(1):71-82, 1986.

The Nursing Assistance was organized by the teachers of Medical-Surgical Nursing of the University of São Paulo (Brasil) School of Nursing. The evaluation was made by both clients-technical, administrative and domestic personnel of the School - and teachers responsible for the services. Both groups mentioned satisfaction with the attendance received or rendered and made suggestions for their improvement. 


\section{ANEXO I \\ UNIVERSIDADE DE SAO PAULO \\ ESCOLA DE ENFERMAGEM \\ DEPARTAMENTO DE ENFERMAGEMM MEDICO-CIRÚRGICA \\ DISCIPLINA ENFERMAGEM MEDICO-CIRURGICA I \\ CENTRO ASSISTENCIAL DE ENFERMAGEM \\ QUESTINARIO PARA AVALIACAO DE ATENDIMENTO}

1. O atendimento do Centro Assistencial de Enfermagem (Consultório) está satisfazendo ao(à) senhor(a) ?

$\operatorname{sim}()$ não $($ )

por quê?

2. Houve pontos no atendimento que o desagradaram?

$\operatorname{sim}($ ) não ( )

por quê?

3. O que você sugere para a melhoria do atendimento no Centro Assistencial de Enfermagem (Consultório)?

- R.:

- P.:

- Curativo:

- Imobilizações:

- Orientações específicas:

- Medicações: V.O.:

I.M.:

E.V.:

S.O.:

- Emergência:

- Fornecimento de medicação:

- Outros:

- Retornos:

- Aulas: assuntos:
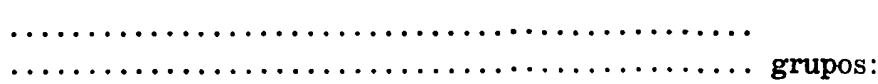

- Palestras: assuntos:
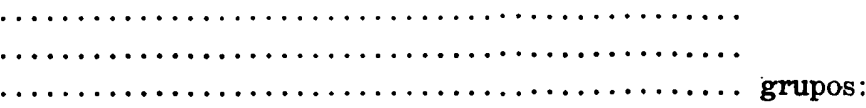

- Reunióes: assuntos:

grupos:

-- Outras atividades: 


\section{ANEXO $\Pi$ \\ UNIVERSIDADE DE SAO PAULO \\ ESCOLA DE ENFERMAGEM \\ CENTRO ASSISTENCIAL DE ENFERMAGEM \\ DISCIPLINA ENFERMAGEM MEDICO-CIRÚRGICA \\ MOVIMENTO ESTATASTICO MENSAL}

Mes:..............

Ano:

- Convocações:

- Primeira consulta:

- Encaminhamentos:

- na EE: Assistência nutricional:

- Enf. Ginec. e Obstétrica:

- Enf. Pediátrica:

- Enf. Psiquiátrica:

- Enf. em Doenças Transmissiveis:

- Enf. em Centro Cirúrgico:

- Outras:

- fora da EE: - Clin. Dermatologica:

- Adolfo Lutz:

- Imunizaçőes:

- Odontologia:

- Oftalmologia:

- Liga Hipertensão:

- H. S. P. E.:

- Serv. Oncologia:

- Saúde do Adulto (C. S.):

- P. S.:

- Atendimentos:

- Pressão Arterial:

- Temperatura:

H.S.P.E. - Hospital do Servidor Público Estadual.

C.S. - Centro de Saúde.

P.S. - Pronto Socorro. 